\title{
Correction to: GPC3 affects the prognosis of lung adenocarcinoma and lung squamous cell carcinoma
}

\author{
Jing Ning ${ }^{1,2 \dagger}$, Shenyi Jiang ${ }^{3 \dagger}$, Xiaoxi Li ${ }^{4}$, Yang Wang ${ }^{1}$, Xuhong Deng ${ }^{1}$, Zhiqiang Zhang ${ }^{5}$, Lijie He ${ }^{5}$, \\ Daqing Wang ${ }^{5^{*}}$ and Youhong Jiang ${ }^{1 *}$
}

\section{Correction to: BMC Pulm Med (2021) 21:199} https://doi.org/10.1186/s12890-021-01549-9

Following publication of the original article [1], it was brought to the authors' attention that the control group and the LUSC group in Fig. 8 had been mislabelled and that the descriptions of $\mathrm{A}$ and $\mathrm{B}$ in the figure legend in Fig. 9 had been reversed, with the result that there were errors in the article content associated with these figures. The figures and the related content have now been corrected in the published article, and the corrected figures and corrected content can be found detailed below:

\begin{abstract}
"Results: The qRT-PCR result showed that GPC3 expression was much lower in the LUSC tissues than that in the control group. IHC results further showed that GPC3 was more negatively expressed than positively expressed in LUSC tissues."
\end{abstract}

\begin{abstract}
Methods
"The paraffin sections of cancer tissues were collected from 10 patients undergoing pulmonary malignant tumour surgery at the Liaoning Cancer Hospital and Institute between June 2018 and June 2020."

\section{Results}

"GPC3 expression was much lower in the LUSC tissues than that in the control group $(P<0.001$, Fig. 8$)$."

"IHC analysis revealed negative staining of GPC3 protein in seven of the ten (70\%) paraffin-embedded LUSC tissues, while positive staining was observed in the remaining cases (three of ten, 30\%) (Fig. 9). This suggests that the low expression of GPC3 may be related to the occurrence of LUSC."

The authors thank you for reading this correction and apologize for any inconvenience caused.
\end{abstract}




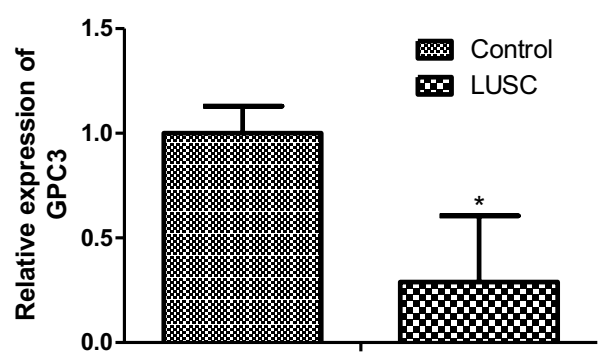

Fig. 8 Relative mRNA expression by quantitative real-time PCR of GPC3 in LUSC compared with control group, ${ }^{*} P<0.05$

A

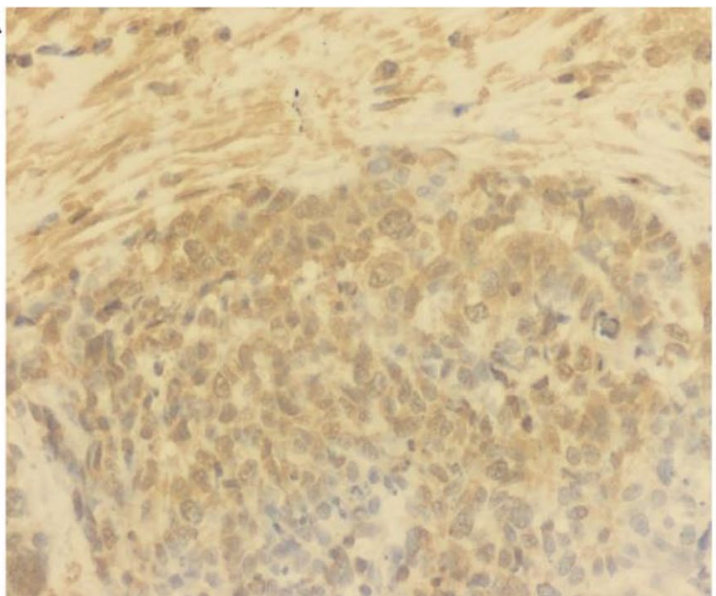

B

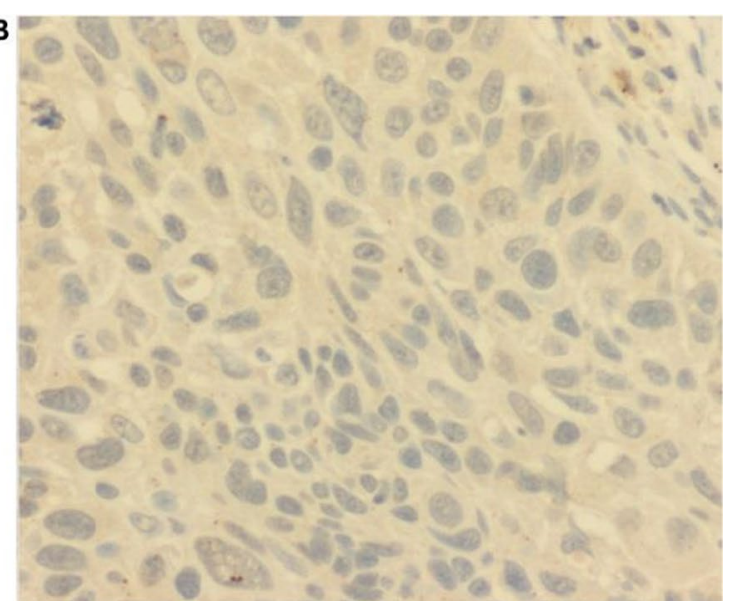

Fig. 9 Representative photos of positive immunohistochemical GPC3 expression in LUSC tissues (A) and negative GPC3 expression in LUSC tissues

\section{Author details}

${ }^{1}$ Molecular Oncology Department of Cancer Research Institution, The First Hospital of China Medical University, Nanjingbei Street, Heping District, Shenyang 110001, Liaoning Province, China. ${ }^{2}$ Department of General Medicine (VIP Ward) and Department of Tumor Supportive and Palliative Medicine, Cancer Hospital of China Medical University, Liaoning Cancer Hospital and Institute, No.44 Xiaoheyan Road, Dadong District, Shenyang 110042, Liaoning Province, China. ${ }^{3}$ Department of General Practice, The First Hospital of China Medical University, Nanjingbei Street, Heping District, Shenyang 110001, Liaoning Province, China. ${ }^{4}$ Central Laboratory, Cancer Hospital of China Medical University, Liaoning Cancer Hospital and Institute, No. 44 Xiaoheyan Road, Dadong District, Shenyang 110042, Liaoning Province, China. ${ }^{5}$ The People's Hospital of Liaoning Province, No.33 Wenyi Road, Shenhe District, Shenyang 110016 , Liaoning Province, China.
Published online: 22 November 2021

\section{Reference}

1. Ning J, Jiang $S$, Li X, Wang $Y$, Deng $X$, Zhang Z, He L, Wang D, Jiang Y. GPC3 affects the prognosis of lung adenocarcinoma and lung squamous cell carcinoma. BMC Pulm Med. 2021;21:199.

\section{Publisher's Note}

Springer Nature remains neutral with regard to jurisdictional claims in published maps and institutional affiliations. 